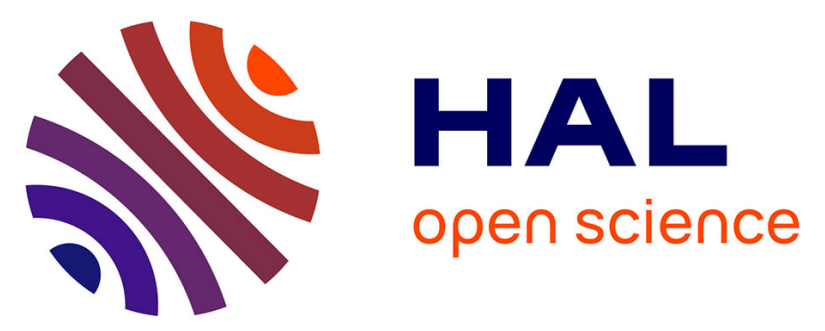

\title{
In depth characterization of Ge-Si core-shell nanowires using X-ray coherent diffraction and time resolved pump-probe spectroscopy
}

Sara Fernandez, Cyril Jean, Eric Charron, Pascal Gentile, Marie-Ingrid Richard, Olivier Thomas, Bernard Perrin, Laurent Belliard

\section{To cite this version:}

Sara Fernandez, Cyril Jean, Eric Charron, Pascal Gentile, Marie-Ingrid Richard, et al.. In depth characterization of Ge-Si core-shell nanowires using X-ray coherent diffraction and time resolved pumpprobe spectroscopy. Journal of Applied Physics, 2019, 126 (20), pp.204304. 10.1063/1.5122263 . hal-02457440

\author{
HAL Id: hal-02457440 \\ https://hal.science/hal-02457440
}

Submitted on 29 Jan 2020

HAL is a multi-disciplinary open access archive for the deposit and dissemination of scientific research documents, whether they are published or not. The documents may come from teaching and research institutions in France or abroad, or from public or private research centers.
L'archive ouverte pluridisciplinaire HAL, est destinée au dépôt et à la diffusion de documents scientifiques de niveau recherche, publiés ou non, émanant des établissements d'enseignement et de recherche français ou étrangers, des laboratoires publics ou privés.

\section{(c)(1)}

Distributed under a Creative Commons Attribution| 4.0 International License 


\title{
In depth characterization of Ge-Si core-shell nanowires using $X$-ray coherent diffraction and time resolved pump-probe spectroscopy
}

\author{
Sara Fernández, ${ }^{1,2}$ Cyril Jean, ${ }^{3}$ Eric Charron, ${ }^{3}$ Pascal Gentile, ${ }^{4}$ Marie-Ingrid Richard,,${ }^{1,2, a)}$ Olivier Thomas, ${ }^{1}$ Bernard Perrin, ${ }^{3}$ and \\ Laurent Belliard ${ }^{3, \text { b) }}$ \\ ${ }^{1}$ Aix Marseille Université, CNRS, Université de Toulon, IM2NP UMR 7334, 13397 Marseille, France \\ ${ }^{2}$ ID01/ESRF, 71 Avenue des Martyrs, CS40220, F-38043 Grenoble Cedex 9, France \\ ${ }^{3}$ Sorbonne Universités, CNRS UPMC Université Paris 06, UMR 7588, Institut des NanoSciences de Paris, F-75005 Paris, France ${ }^{4}$ CEA, INAC- \\ SP2M, SINAPS, F-38000 Grenoble, France \\ a)mrichard@esrf.fr \\ b)Laurent.Belliard@upmc.fr
}

\begin{abstract}
We report on the ultrafast vibrational response of single Ge-Si core-shell nanowires obtained by epitaxial growth and investigated by femtosecond transient reflectivity and coherent x-ray diffraction measurements. The oscillations of the sample reflectivity are correlated with the fundamental breathing mode for wires with a diameter ranging from 150 to $350 \mathrm{~nm}$ and compared with solutions of the Navier equation. Taking advantage of a free standing geometry, we are able to get a mechanical quality factor of higher than 80 . Coupling electron microscopy and pump and probe investigations with a very high spectral resolution performed on the same wire, we demonstrate that both shell and core diameter fluctuations are revealed and quantified. X-ray coherent diffraction measurements on individual nanowires evidence changes in the Ge-core diameter and different strain states along a single structure.
\end{abstract}

\section{INTRODUCTION}

Discrete vibrational modes, confined in semiconductor coreshell nano-objects, remain rarely investigated using a standard pump and probe scheme ${ }^{1}$ compared to metal-insulating two-component nano-objects, ${ }^{2}$ metal-metal, ${ }^{3-8}$ or mono-component, ${ }^{9-14}$ whereas they appear as good candidates for a wide variety of applications. In phononic and photonic engineering, semiconductor nanowires are used to design single photon sources controlled by surface acoustic waves. ${ }^{15}$ They are also good candidates for developing new thermoelectric devices. ${ }^{16-18}$ Except for a recent investigation focused on in situ silver shell coating on a gold nanocylinder, ${ }^{19}$ core-shell or alloy systems have only been studied on particle assemblies using an ultrafast acoustics approach, ${ }^{3,7}$ leading to an inhomogeneous broadening in the frequencies of the detected eigenmodes. In addition, these studies are restricted to metals with low acoustic contrasts, making any characterization a challenge. However, different behaviors have been already observed: associated with a poor shell adhesion, the vibration could be confined in the core or in the shell, ${ }^{2,20,21}$ the resonance modes could be reproduced using an effective model describing the system as an alloy, or in good agreement with a matrix modeling the core-shell architecture. ${ }^{7}$ In this context, the acoustic study in individual core-shell semiconductor systems represents a real breakthrough. In this article, vibrational modes in single Ge-Si core shell nanowires, with a diameter of around $150-300 \mathrm{~nm}$ are monitored in the time domain using ultrafast optical pump and probe spectroscopy. Taking advantage of a free standing geometry, suppressing the energy relaxation channel through the substrate across the surface of contact, we were able to get nanoresonators to exhibit high quality factors. ${ }^{7}$ A semianalytical model is used to describe the breathing mode of nanowires, and the silicon shell thickness is deduced, thanks to the good 
spectral accuracy. Complementary X-ray coherent diffraction measurements were also applied. ${ }^{22,23}$ They confirm a variation in the diameter of the core and reveal a strain and lattice tilt along the nanowire axis.

\section{EXPERIMENTAL}

\section{A. Pump-probe spectroscopy}

First, vertical Ge nanowires are epitaxially grown by chemical vapor deposition on a $\mathrm{Ge}(111)$ substrate. In the second step, $\mathrm{Si}$ is deposited to get the shell [Fig. 1(a)]. The core-shell nanowires are finally separated from the germanium substrate using an ultrasonic bath and dispersed over a silicon wafer preliminarily structured with periodic pyramidal trenches [Fig. 1(b)]. Such a geometry ensures a better acoustic confinement, in other words, the damping rate of the elastic response is reduced. Trench numbering allows one to locate the same nanowire for elastic measurement as well as for scanning electron microscope investigation. The nanowire diameter which is not constant along the axis [Fig. 1(b)] is thus determined. In the following, we demonstrate that measuring the dynamic response along the axis sheds light on the real shell thickness. Our pump and probe experimental setup working in the reflection geometry is described in detail elsewhere. ${ }^{24,25}$ A mode-locked Ti:sapphire (MAI TAI Spectra) laser source operating at $800 \mathrm{~nm}$ is used to thermally excite and optically detect the nanowire's vibrational modes. A twocolor scheme is performed by doubling the pump frequency $(\lambda=400 \mathrm{~nm})$ with a nonlinear crystal $(\mathrm{BBO})$ to avoid scattered light coming from the pump. The blue pump power is fixed to $300 \mu \mathrm{W}$ with a red probe power equal to $30 \mu \mathrm{W}$. For such a low optical power, the reflectivity is measured by an avalanche photodiode and analyzed with a lock-in amplifier. A 12 ns maximum

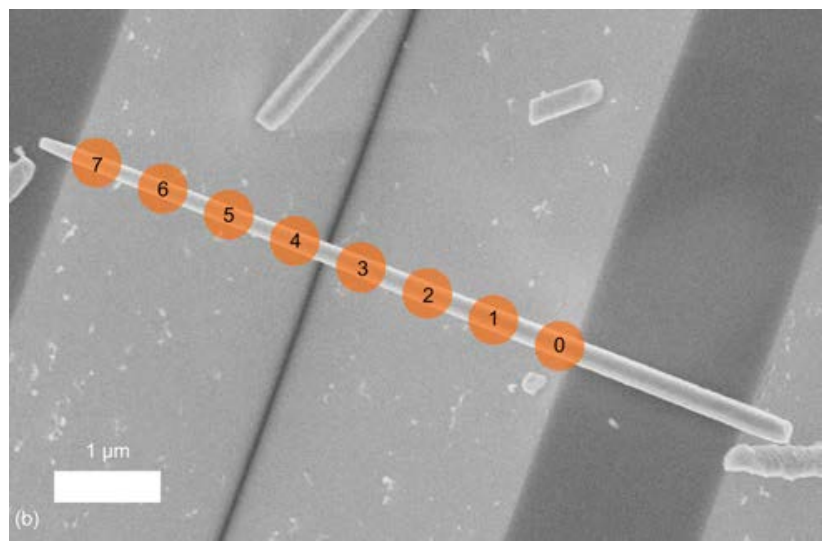

FIG. 1. (a) The scanning electron microscope image of the as-grown Ge-Si core-shell nanowires. (b) Single nanowire, crossing a pyramidal trench fabricated by lithography and anisotropic silicon etching, with a total diameter close to $150 \mathrm{~nm}$. The orange labels correspond to the localizations where the pump and probe measurements have been carried out.

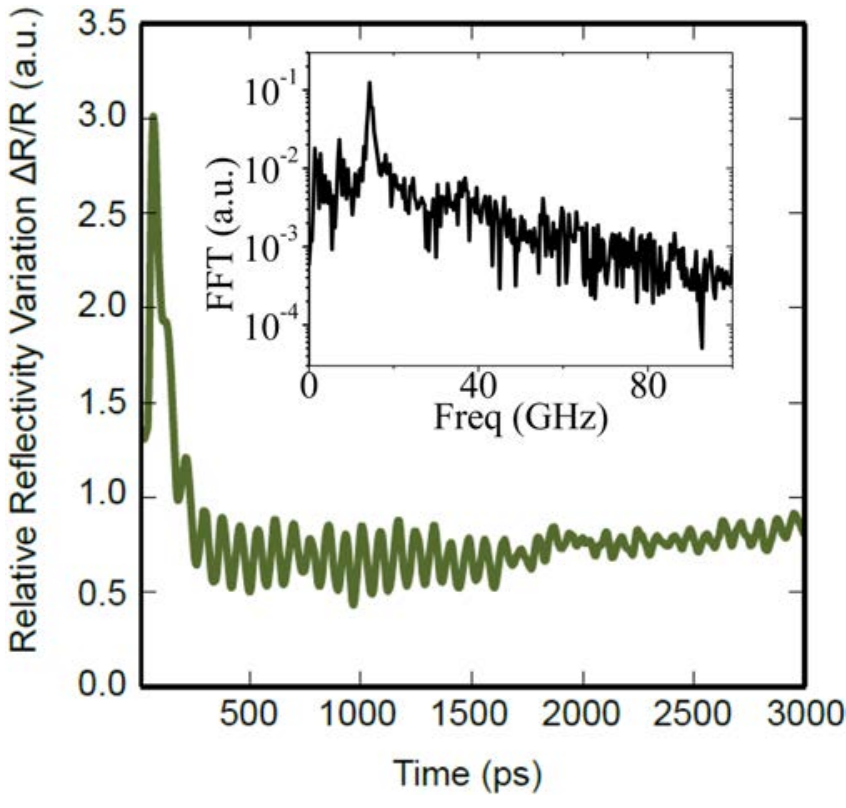

FIG. 2. Transient reflectivity obtained on a free standing Ge-Si core-shell nanowire with a total diameter close to $250 \mathrm{~nm}$. Inset: Fast Fourier Transform (FFT) of the experimental signal showing a sharp peak around $14 \mathrm{GHz}$.

pump-probe time delay is achieved using a mobile reflector mounted on a translation stage. Both pump and probe beams are focused with a microscope objective with a numerical aperture of 0.9 , fixed on a piezoelectric stage to scan the elastic response along the nanowire axis.

A typical transient reflectivity measured on a suspended single nanowire is given in Fig. 2. Except for the sharp electronic signature in the first picoseconds, the signal is mainly dominated by an oscillating response close to $14 \mathrm{GHz}$. Additional higher frequency components could be detected but due to their much lower magnitude, we have neglected them in the following. This mentioned frequency component is correlated to the nanowire size, namely, blueshifted for smaller diameters. As usually reported, ${ }^{26}$ femtosecond laser excitation pulses absorbed in nanoobjects induce modal selection revealing specifically the breathing mode and their harmonics in infinitely long nanowires, characterized by a pure radial displacement. Cubic Ge and Si materials exhibit a low anisotropic Zener index close to 1.6, then the frequency changes linked to specific crystalline orientation are expected to be small. To quantify this effect, finite element simulations, including cubic elastic tensor, have been performed in a pure silicon nanowire for all the nanowire orientations and are reported in Fig. 3. The associated frequency variation reaches only $0.5 \mathrm{GHz}$ for the breathing mode centered at $29 \mathrm{GHz}$. As long as the nanowire is supported on a substrate, such small variations cannot be detected due to their low quality factor. Taking advantage of a better elastic confinement in free suspended nanowires, such an anisotropic feature could be 

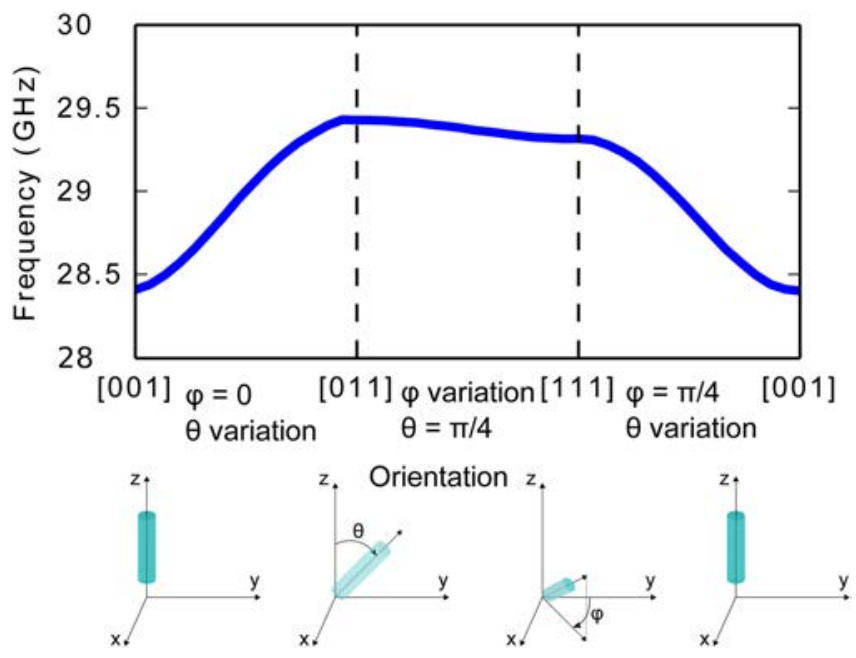

FIG. 3. Simulated frequency variation of the first radial breathing mode in a pure silicon nanowire with a total diameter of $200 \mathrm{~nm}$, for different nanowires orientations. $C_{11}=166 \mathrm{GPa}, C_{12}=64 \mathrm{GPa}$, and $C_{44}=80 \mathrm{GPa}$. For the $\mathrm{Ge}$ core, this curve is similar with lower frequency response.

revealed but remains smaller than the effect of the fluctuating shell thickness as discussed in the following. Consequently, we only consider their isotropic elastic constants. The vibrational mode frequency is generally obtained by solving the Navier equation for the isotropic material. The standard resolution is based on the Lame potentials method for isotropic material ${ }^{27-29}$ and applying the free boundary conditions. Finite element simulations, which were carried out to quantify the effect of the real hexagonal shape, show a difference of only $2-3 \mathrm{GHz}$ in the studied diameter range. This discrepancy has the same magnitude than the accuracy of the frequency determination on oscillations exhibiting a quality factor around 20, then the cylindrical hypothesis will be considered in the following to reach a semianalytical model. For a pure cylindrical nanowire, the breathing eigenmode equation is given by

$$
2\left(\frac{\mathrm{c}_{\mathrm{T}}}{\mathrm{c}_{\mathrm{L}}}\right)^{2} \mathrm{~J}_{1}\left(\frac{\omega a}{\mathrm{c}_{\mathrm{L}}}\right)=\frac{\omega a}{\mathrm{c}_{\mathrm{L}}} \mathrm{J}_{0}\left(\frac{\omega a}{\mathrm{c}_{\mathrm{L}}}\right)
$$

with $a$ being the nanowire radius, $\omega$ the pulsation, and $c_{L}, c_{T}$ the longitudinal and transverse sound velocity, respectively, and $\mathrm{J}_{\mathrm{i}}$ the Bessel functions. For infinitely long core shell nanowires, taking into account a zero stress at the core-shell boundary, the axisymmetric longitudinal modes $f_{\mathrm{L}(0, \mathrm{j})}^{(\mathrm{co} / \mathrm{sh})}$ are given by ${ }^{30}$

$$
\left|\begin{array}{ccc}
d_{11} & d_{12} & d_{13} \\
d_{21} & d_{22} & d_{23} \\
0 & d_{32} & d_{33}
\end{array}\right|=0
$$

with

$$
\begin{aligned}
& d_{11}=\mathrm{J}_{1}\left(x_{j}^{(\mathrm{sh})} \gamma f\right), \\
& d_{12}=-\mathrm{J}_{1}\left(x_{j}^{(\mathrm{sh})} \gamma\right), \\
& d_{13}=-\mathrm{Y}_{1}\left(x_{j}^{(\mathrm{sh})} \gamma\right), \\
& d_{21}=\frac{C_{11}^{(\mathrm{co})}}{a^{(\mathrm{co})}}\left[x_{j}^{(\mathrm{sh})} \gamma f \mathrm{~J}_{0}\left(x_{j}^{(\mathrm{sh})} \gamma f\right)-2\left(\frac{\mathrm{c}_{\mathrm{T}}^{(\mathrm{cos})}}{\mathrm{c}_{\mathrm{L}}^{(\mathrm{co})}}\right)^{2} \mathrm{~J}_{1}\left(x_{j}^{(\mathrm{sh})} \gamma f\right)\right], \\
& d_{22}=\frac{C_{11}^{(\mathrm{sh})}}{a^{(\mathrm{co})}}\left[-x_{j}^{(\mathrm{sh})} \gamma \mathrm{J}_{0}\left(x_{j}^{(\mathrm{sh})} \gamma\right)+2\left(\frac{\mathrm{c}_{\mathrm{T}}^{(\mathrm{sh})}}{\mathrm{c}_{\mathrm{L}}^{(\mathrm{sh})}}\right)^{2} \mathrm{~J}_{1}\left(x_{j}^{(\mathrm{sh})} \gamma\right)\right], \\
& d_{23}=\frac{C_{11}^{(\mathrm{sh})}}{a^{(\mathrm{co})}}\left[-x_{j}^{(\mathrm{sh})} \gamma \mathrm{Y}_{0}\left(x_{j}^{(\mathrm{sh})} \gamma\right)+2\left(\frac{\mathrm{c}_{\mathrm{T}}^{(\mathrm{sh})}}{\mathrm{c}_{\mathrm{L}}^{(\mathrm{sh})}}\right)^{2} \mathrm{Y}_{1}\left(x_{j}^{(\mathrm{sh})} \gamma\right)\right], \\
& d_{32}=\frac{C_{11}^{(\mathrm{sh})}}{a^{(\mathrm{sh})}}\left[-x_{j}^{(\mathrm{sh})} \mathrm{J}_{0}\left(x_{j}^{(\mathrm{sh})}\right)+2\left(\frac{\mathrm{c}_{\mathrm{T}}^{(\mathrm{sh})}}{c_{\mathrm{L}}^{(\mathrm{sh})}}\right)^{2} \mathrm{~J}_{1}\left(x_{j}^{(\mathrm{sh})}\right)\right], \\
& d_{33}=\frac{C_{11}^{(\mathrm{sh})}}{a^{(\mathrm{sh})}}\left[-x_{j}^{(\mathrm{sh})} \mathrm{Y}_{0}\left(x_{j}^{(\mathrm{sh})}\right)+2\left(\frac{c_{\mathrm{T}}^{(\mathrm{sh})}}{c_{\mathrm{L}}^{(\mathrm{sh})}}\right)^{2} \mathrm{Y}_{1}\left(x_{j}^{(\mathrm{sh})}\right)\right],
\end{aligned}
$$

where $a^{(\mathrm{sh})}$ is the radius of the nanowire and $a^{(\mathrm{co})}$ is the core radius. $c_{\mathrm{L}}^{(\mathrm{co})}$ and $c_{\mathrm{T}}^{(\mathrm{co})}$ are the longitudinal and transverse sound velocities of the isotropic material of the core; $c_{\mathrm{L}}^{(\mathrm{sh})}$ and $c_{\mathrm{T}}^{(\mathrm{sh})}$ are the longitudinal and transverse speed of sound of the isotropic material of the shell. $C_{11}^{(\mathrm{sh})}$ and $C_{11}^{(\mathrm{co})}$ are, respectively, the elastic constant of the materials constituting the shell and the core. Let us write $x_{j}^{(\mathrm{sh})}=2 \pi f_{\mathrm{L}(0, \mathrm{j})}^{(\mathrm{so})} a^{(\mathrm{sh})} / \mathrm{c}_{\mathrm{L}}^{(\mathrm{sh})}, \quad \gamma=\mathrm{c}_{\mathrm{L}}^{(\mathrm{sh})} / \mathrm{c}_{\mathrm{L}}^{(\mathrm{co})}$, and $f=a^{(\mathrm{co})} / a^{(\mathrm{sh})}$. The first radial breathing mode is the solution $j=2$.

The normalized breathing mode period vs the nanowire diameter is plotted in Fig. 4 for different shell thicknesses. Only the nanowires exhibiting a quality factor larger than 20 have been selected. The quality factor $Q$ of the resonance is given by $\pi^{\star} f^{\star} \tau$, with $f$ being the frequency of resonance and $\tau$ the exponential time decay. As expected for constant nanowire diameter, the period of the breathing mode increases for smaller shell thickness due to smaller elastic constants of the Ge core. Additionally, the change induced by the shell is larger for smaller diameter. The error bars have been estimated using the $\tau$ parameter.

Coupling SEM investigations to measure the diameter at different locations along the nanowire axis placed over a trench with picosecond acoustic measurements performed at the same location, we can superimpose the experimental results in Fig. 4. Special care has been paid to extract errors bars taking into account the quality factor of the observed resonances. Two families can be clearly distinguished, the first one is characterized by a poor accuracy of the breathing mode frequency, due to huge intrinsic damping rate. This family has been excluded from fine analysis. The second one exhibits a very small dispersion of the period of vibration. Among the nanowires belonging to the second family, some of them exhibit resonance frequencies which could not be reproduced by a single simulated curve, highlighting both 


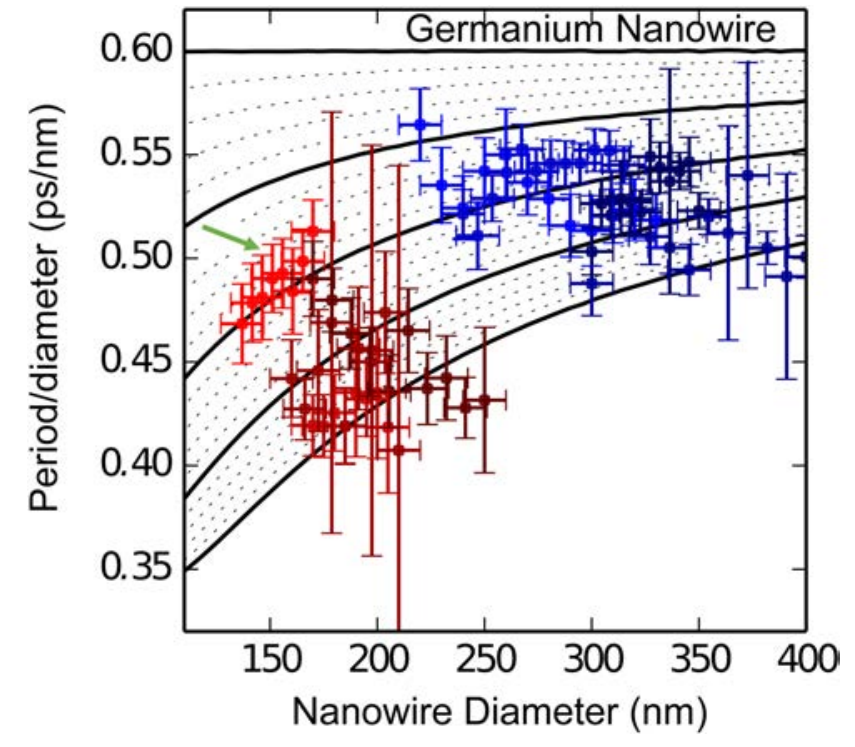

FIG. 4. Normalized breathing mode period plotted against the nanowire total diameter. Bold black lines correspond to shell thicknesses equal to $0,10,20$, 30 , and $40 \mathrm{~nm}$ from top to bottom. Dashed lines have been calculated with a step of $2 \mathrm{~nm}$. The elastic constants and density values used are $E_{\mathrm{Si}}=169 \mathrm{GPa}, E_{\mathrm{Ge}}=132 \mathrm{GPa}, v_{\mathrm{Si}}=0.22, v_{\mathrm{Ge}}=0.19, \rho_{\mathrm{Si}}=2330 \mathrm{~kg} / \mathrm{m}^{3}$, $\rho_{\mathrm{Si}}=5320 \mathrm{~kg} / \mathrm{m}^{3}$ for the Young modulus, the Poisson ratio, and density, respectively. Colored data have been recorded along the axis of different nanowires with a nominal total diameter around 150 and $300 \mathrm{~nm}$, each color represents one nanowire response. The green arrow underlines measurements performed on label 0-7 on a nanowire exhibiting a constant shell thickness and a high quality factor.

fluctuation of the core and shell size. Inversely, we can notice that some wires express an elastic behavior in good agreement with an assumption based on a constant shell thickness along the axis (see the arrow in Fig. 4). The change of the total diameter is mainly associated with the core's diameter variation. Selecting the latter nanowires, we can estimate the fluctuation of the silicon shell thickness deposited on the germanium core. Such experiments, realized over 56 nanowires coupling diameter investigation performed by electron microscopy and ultrafast spectroscopy, are summarized in Fig. 5. Only the nanowires exhibiting quality factor larger than 20 have been selected. The fluctuation of the shell thickness is large from 10 up to $45 \mathrm{~nm}$, with a modal value equal to $23.1 \mathrm{~nm}$ (see Fig. 5) in good agreement with the nominal value of $20 \mathrm{~nm}$.

\section{B. X-ray coherent diffraction}

In order to get a detailed understanding of the crystallographic structure of these nanowires, X-ray coherent diffraction experiments were performed on single $\mathrm{Ge}$-Si core-shell nanowires at the ID01 beamline at the ESRF (Grenoble, France). Since the ID01 beamline provides a nano-focused beam, of the order of the nanowire diameter, they can be studied either as grown or after detaching them from the Ge-substrate and deposited on the Si-trenches. The latter procedure may induce crystallographic

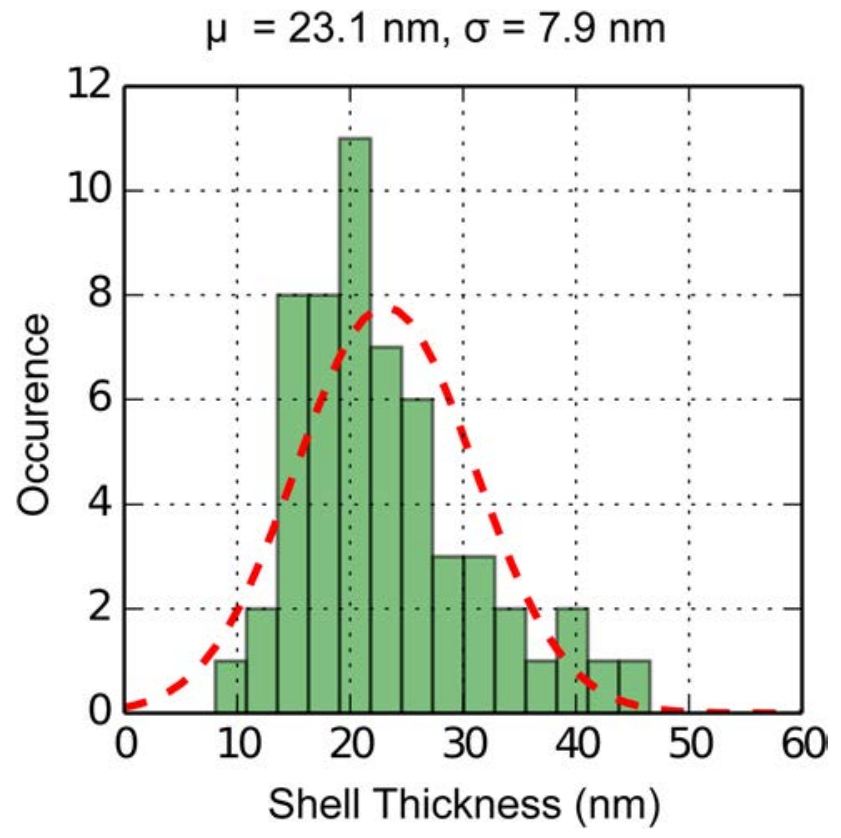

FIG. 5. Estimated shell thickness distribution histogram. Adjusted in red by a normal distribution, with a standard deviation $\sigma=7.9 \mathrm{~nm}$ centered in $\mu=23.1 \mathrm{~nm}$. The median quality factor is greater than 20 .

defects in the structure so that it is preferable to use a SEM-FIB manipulator to isolate one of the wires, as explained elsewhere ${ }^{31}$ and shown in Fig. 6, in order to be studied by X-ray diffraction. In this way, it is possible to analyze the crystal structure of a single nanowire and localize defects or map elastic strain.

The synchrotron experiment was performed at $8 \mathrm{keV}$, the coherent X-ray beam being selected using upstream slits and then

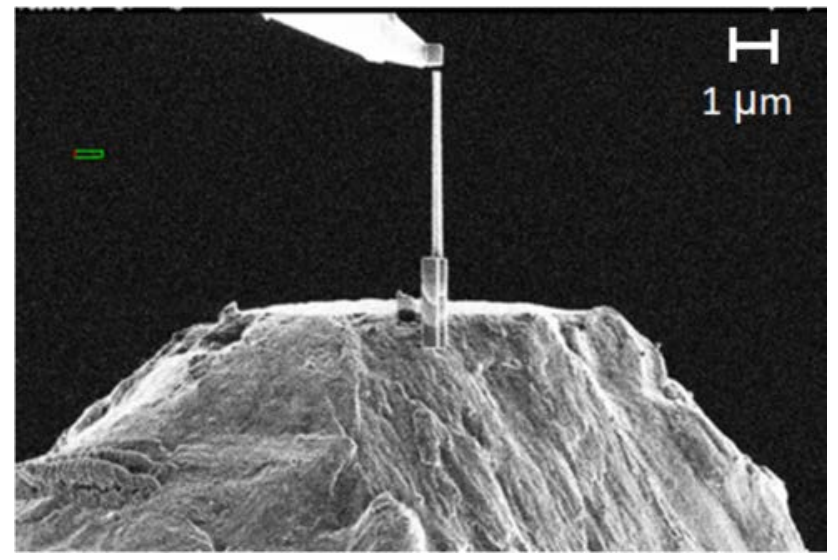

FIG. 6. SEM image showing how the nanowire (NW) is isolated using a SEM-FIB manipulator. The NW is glued on a Cu tip with carbon in the upright position. 
focused with a Fresnel zone plate to a spot size of $100 \times 400 \mathrm{~nm}^{2}$ on the sample, which consisted of a nanowire with a core diameter of about $200 \mathrm{~nm}$, a shell of $50 \mathrm{~nm}$ (from growth parameters), and an exploitable length of $7 \mu \mathrm{m}$. The intensity distribution around the $111 \mathrm{Ge}$ Bragg reflection was measured in coplanar diffraction geometry, with a 2D MAXIPIX detector placed in the far field. At the working energy, $111 \mathrm{Ge}$ and Si Bragg reflections are accessible at a scattering angle of $2 \theta_{B}=27.44^{\circ}$ and $28.63^{\circ}$, respectively. Rocking curves at different positions along the length of the nanowire were performed around the $111 \mathrm{Ge}$ Bragg reflection with a step size of $\Delta \theta=0.005^{\circ}$. The recorded diffraction patterns, taken every $800 \mathrm{~nm}$ along the wire, are displayed in Fig. 7.

The diffraction patterns are characterized by six streaks that correspond to a hexagonal cross section of the wire, despite the fact that a circular section has been assumed in the vibrational analysis. One-dimensional cuts along the streaks show a modulated intensity whose periodicity relates to the well-defined crystalline facets of the wire as $d=\frac{2 \pi}{\Delta Q}$, where $d$ is the diameter of the nanowire and $\Delta Q$ the distance between interference fringes in the diffraction pattern along the streak direction. The thickness fringes visible in Fig. 7 lead to a $220 \mathrm{~nm}$ diameter that varies along the length: a difference of about $45 \mathrm{~nm}$ was found between the upper and lower positions where the measurements were performed, the wire being thicker at the bottom. No signal from the shell was detected either at the Si

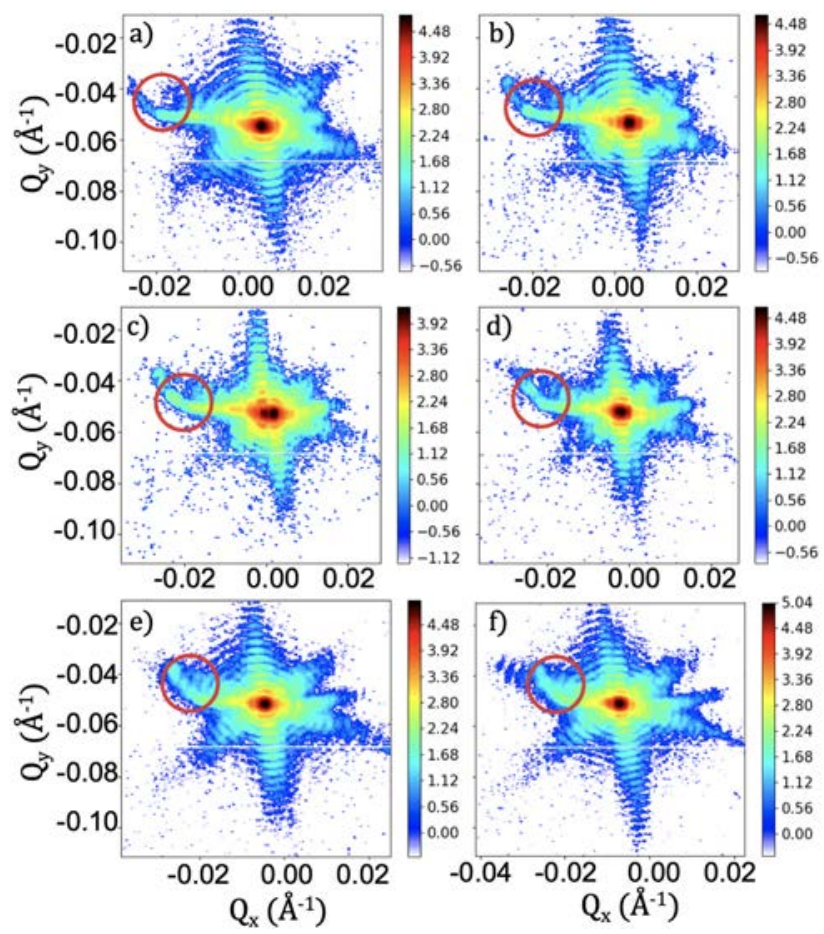

FIG. 7. Diffraction patterns recorded around the $111 \mathrm{Ge}$ Bragg reflection at different positions (every $800 \mathrm{~nm}$ ) along the length of a single nanowire, from top to bottom. The red circles show the spotty-streak from other parts of the wire illuminated by the footprint of the X-ray beam. bulk position, probably due to its low thickness. In order to investigate the Si-shell, independent TEM studies of NWs grown in the same fashion but with different sizes were performed. The cross section of one of them is shown in Fig. 8.

In addition to the thickness variation of the Ge determined from the diffraction patterns, a spotty-streak (circled in Fig. 7) is visible all along the wire. This streak is believed to arise from other illuminated parts of the nanowire, which would be illuminated by the large footprint of the X-ray beam on the sample and that would have, therefore, different lattice misorientations because of bending or torsion (see below).

The centrosymmetry of the diffraction patterns may correspond to a free or weakly strained Ge core. However, the strain is not homogeneous along the wire: one may notice a splitting of the diffraction peak in Fig. 7(c) or a double streak in Figs. 7(d) and 7(f). The strain evolution within the wire can be measured taking as reference, for instance, the position of the Ge Bragg peak in the detector at the bottom of the wire [Fig. 7(f)]

$$
\epsilon(\%)=\frac{a-a_{r e f}}{a_{r e f}} \times 100
$$

where $a$ is the lattice parameter at any given position and $a_{r e f}$ is the reference one. The calculated strain is displayed in Fig. 9. The nanowire exhibits a maximal compressive strain at the center of the wire of $\Delta \epsilon=-0.018 \%$.

In addition, if one defines the orientation of the atomic planes at this reference position as "normal orientation," the angular

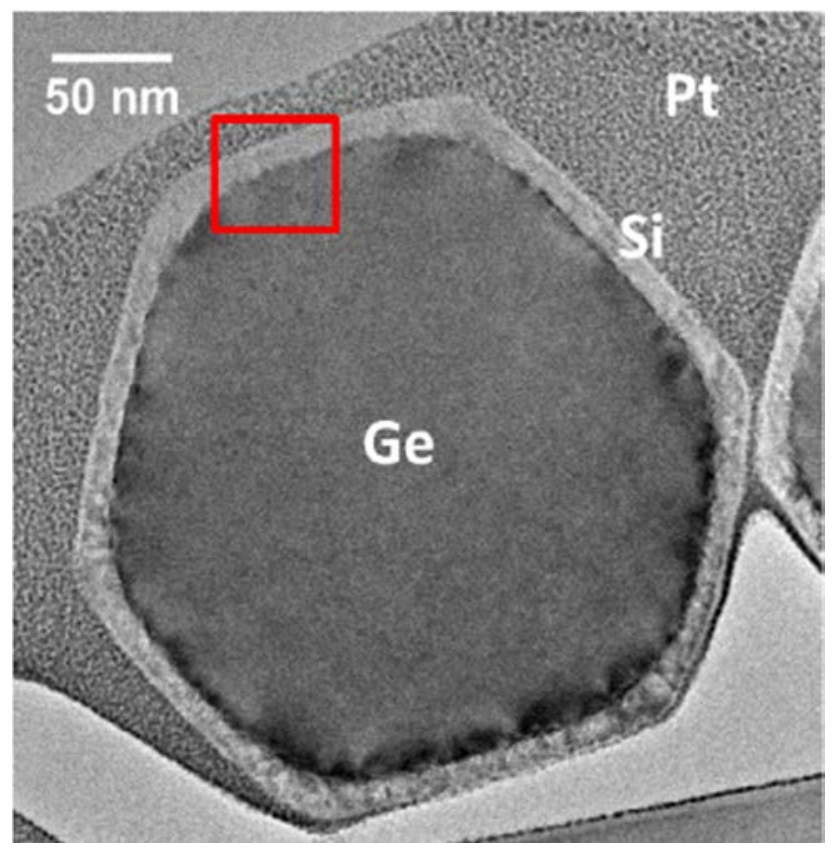

FIG. 8. TEM image showing the core and shell of the Ge-Si nanowire and its hexagonal cross section. 


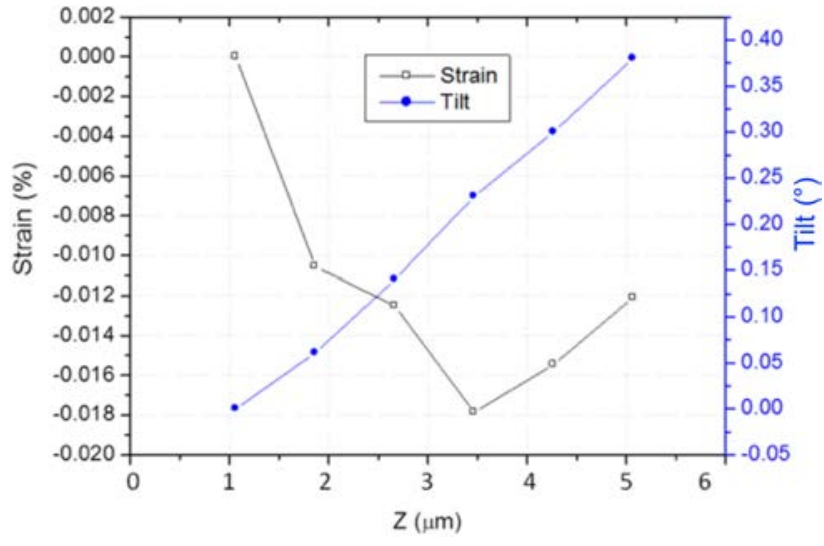

FIG. 9. Strain and tilt of a single nanowire, as a function of its length.

deviation at the other positions along the length of the wire with respect to the reference can be extracted. The difference in tilt of the crystallographic planes, usually referred as "lattice tilt," can be determined as follows:

$$
\left.\alpha=\arccos \frac{\vec{Q}_{r e f} \cdot \vec{Q}}{\left\|\vec{Q}_{r e f}\right\|\|\vec{Q}\|}\right),
$$

where $\vec{Q}_{\text {ref }}$ and $\vec{Q}$ are the scattering vectors at the reference and investigated position, respectively. The results are plotted in Fig. 9, where a linear increase of the tilt is observed, yielding a $0.4^{\circ}\left(=\alpha_{1}\right)$ rotation over $4 \mu \mathrm{m}\left(=h_{1}\right)$ along the wire. The observed linear increase in tilt corresponds to a constant curvature and bending radius, $R$. From Ref. 32, $R$ is given by $R \sim h_{1} / 2 \tan \left(\alpha_{1}\right)=0.29 \mathrm{~mm}$.

A similar experiment could be performed on Si-Ge nanowires lying on silicon, as depicted in Fig. 10. The measurements we performed in this configuration show, however, that the nanowires in

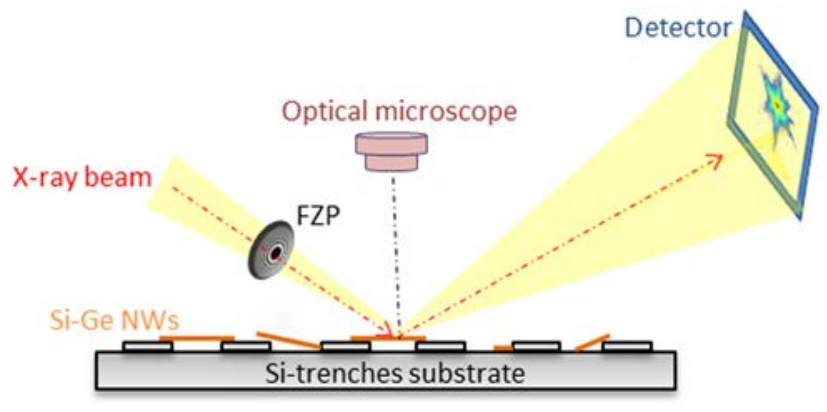

FIG. 10. Sketch of the X-ray diffraction setup for the characterization of individual Si-Ge NWs lying on Si. The coherent beam is focused by a Fresnel Zone Plate (FZP) and impinges onto the NW at the 224 Ge Bragg angle. The diffracted intensity is collected by a $2 \mathrm{D}$ detector.

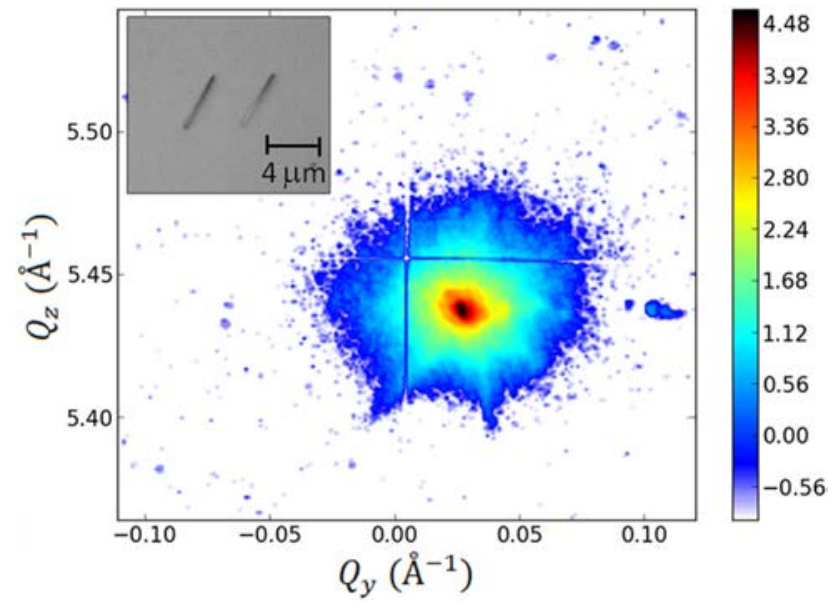

FIG. 11. Diffraction pattern around the $224 \mathrm{Ge}$ Bragg reflection of the Si-Ge nanowire lying on a Si substrate. The measured wire is shown in the inset, being the one of the right.

contact with the substrate are highly strained, as visible in Fig. 11, where the diffracted signal around the $\mathbf{2 2 4}$ Bragg reflection of one nanowire is displayed. In this diffraction pattern, the form factor of the wire is barely visible, the strain being too high to preserve the six streaks arising from the hexagonal Ge core.

It is worth mentioning that the nanowires were observed to lie on one of their $\{112\}$ crystallographic facets when deposited on a $\mathrm{Si}$ substrate, but they might be slightly rotated. This is observed in the optical image displayed in the inset of Fig. 11, where a color change is evidenced. When the wires are suspended between the trenches, the measurement turns out to be difficult because of the tilt. It has been observed, however, that in the case of metallic nanowires, which lie on larger facets, the manipulation does not induce any defect in the suspended area, and strained areas are localized at the contact points with the substrate. ${ }^{33}$

\section{CONCLUSION}

In conclusion, the ultrafast vibrational response of single Ge-Si core-shell nanowires has been demonstrated and analyzed using a semianalytical approach. The identification of different elastic responses highlights the value of individual studies over ensemble measurements. Time resolved elastic measurements coupled with electron microscopy investigations give us the opportunity to identify nanowires exhibiting both shell and core diameter fluctuation in the same batch. The latter had been studied by means of X-ray coherent diffraction measurements, which showed a difference in diameter of $45 \mathrm{~nm}$ over a length of $5 \mu \mathrm{m}$ in a single nanostructure, therefore, in agreement with the fluctuations from the acoustic measurements. Moreover, important variations of strain and crystallographic tilt are evidenced in the recorded diffraction patterns. Up until now, no clear evidence has been reported on the correlation between such inhomogeneous strain distribution and vibrational response. Further investigations would benefit from other coupled nondestructive 
investigations such as simultaneous pump-probe spectroscopy. Such an inhomogeneous strain distribution revealed by X-ray diffraction may have a weak influence on the dynamic response across the frequency response or the mean free path.

First, the residual strain slightly modified the elastic constant, and then a frequency change of the acoustic vibration modes can be expected. Nevertheless, this effect is much lower than the variation induced by the fluctuation of the nanowire diameter, as reported in Fig. 4 and observed in SEM pictures. In order to achieve a relevant correlation between strain distribution and the frequency of vibration, nanowires with a constant diameter may be more appropriate. Moreover, even if the free standing geometry allows one to increase the quality factor of the resonators, this measured attenuation is also strongly related to the size, the guided modes, and the intrinsic attenuation. All these phenomena must be simultaneously taken into account and require specific experiments at different temperatures on nanowires of a constant diameter.

\section{ACKNOWLEDGMENTS}

We thank the ID01-ESRF beamline staff for excellent support during the experiment.

\section{REFERENCES}

${ }^{1}$ T. D. Krauss and F. W. Wise, Phys. Rev. Lett. 79, 5102 (1997).

${ }^{2}$ D. Mongin, V. Juvé, P. Maioli, A. Crut, N. Del Fatti, F. Vallée, A. Sánchez-Iglesias, I. Pastoriza-Santos, and L. M. Liz-Marzán, Nano Lett. 11, 3016 (2011).

${ }^{3}$ M. F. Cardinal, D. Mongin, A. Crut, P. Maioli, B. Rodríguez-Gonzàlez, J. Pérez-Juste, L. M. Liz-Marzàn, N. Del Fatti, and F. Vallée, J. Phys. Chem. Lett. 3, 613 (2012).

${ }^{4}$ H. Petrova, C.-H. Lin, M. Hu, J. Chen, A. R. Siekkinen, Y. Xia, J. E. Sader, and G. V. Hartland, Nano Lett. 7, 1059 (2007).

${ }^{5}$ J. H. Hodak, A. Henglein, and G. V. Hartland, J. Phys. Chem. B 104, 5053 (2000).

${ }^{6}$ L. Wang, A. Kiya, Y. Okuno, Y. Niidome, and N. Tamai, J. Chem. Phys. 134, 054501 (2011).

7T. Stoll, P. Maioli, A. Crut, J. Burgin, P. Langot, M. Pellarin, A. Sánchez-Iglesias, B. Rodríguez-González, L. M. Liz-Marzán, N. Del Fatti, and F. Vallée, J. Phys. Chem. C 119, 1591 (2015).

8. E Sader, G. V. Hartland, and P. Mulvaney, J. Phys. Chem. B 106, 1399 (2002).

${ }^{9}$ V. Juvé, A. Crut, P. Maioli, M. Pellarin, M. Broyer, N. Del Fatti, and F. Vallée, Nano Lett. 10, 1853 (2010).
${ }^{10}$ T. A. Major, A. Crut, B. Gao, S. S. Lo, N. Del Fatti, F. Vallée, and G. V. Hartland, Phys. Chem. Chem. Phys. 15, 4169 (2013).

${ }^{11}$ L. Belliard, T. W. Cornelius, B. Perrin, N. Kacemi, L. Becerra, O. Thomas, M. Toimil-Molares, and M. Cassinelli, J. Appl. Phys. 114, 193509 (2013).

${ }^{12}$ J. Burgin, P. Langot, N. Del Fatti, F. Vallée, W. Huang, and M. A. El-Sayed, J. Phys. Chem. C 112, 11231 (2008).

${ }^{13}$ C. Jean, L. Belliard, L. Becerra, and B. Perrin, Appl. Phys. Lett. 107, 193103 (2015).

${ }^{14}$ C. Jean, L. Belliard, T. W. Cornelius, O. Thomas, M. E. Toimil-Molares, M. Cassinelli, and B. Perrin, J. Phys. Chem. Lett. 5, 4100 (2014).

${ }^{15}$ A. Hernández-Mínguez, M. Möller, S. Breuer, C. Pfüller, C. Somaschini, S. Lazić, O. Brandt, A. García-Cristóbal, M. M. de Lima, A. Cantarero, L. Geelhaar, H. Riechert, and P. V. Santos, Nano Lett. 12, 252 (2011).

${ }^{16}$ A. I. Hochbaum, R. Chen, R. D. Delgado, W. Liang, E. C. Garnett, M. Najarian, A. Majumdar, and P. Yang, Nature 451, 163 (2008).

${ }^{17}$ M. Hu, K. P. Giapis, J. V. Goicochea, X. Zhang, and D. Poulikakos, Nano Lett. 11, 618 (2011).

${ }^{18}$ M. C. Wingert, Z. C. Y. Chen, E. Dechaumphai, J. Moon, J.-H. Kim, J. Xiang, and R. Chen, Nano Lett. 11, 5507 (2011).

${ }^{19}$ K. Yu, J. E. Sader, P. Zijlstra, M. Hong, Q. H. Xu, and M. Orrit, Nano Lett. 14, 915 (2014).

${ }^{20}$ C. Guillon, P. Langot, N. Del Fatti, F. Vallée, A. S. Kirakosyan, T. V. Shahbazyan, T. Cardinal, and M. Treguer, Nano Lett. 7, 138 (2007).

${ }^{21}$ D. A. Mazurenko, X. Shan, J. C. P. Stiefelhagen, C. M. Graf, A. van Blaaderen, and J. I. Dijkhuis, Phys. Rev. B 75, 161102 (2007).

${ }^{22}$ D. Dzhigaev, A. Shabalin, T. Stankevič, U. Lorenz, R. P. Kurta, F. Seiboth, J. Wallentin, A. Singer, S. Lazarev, O. M. Yefanov, M. Borgström, M. N. Strikhanov, L. Samuelson, G. Falkenberg, C. G. Schroer, A. Mikkelsen, R. Feidenhans'l, and I. A. Vartanyants, J. Opt. 18, 064007 (2016).

${ }^{23}$ J. Wallentin, D. Jacobsson, M. Osterhoff, M. T. Borgström, and T. Salditt, Nano Lett. 17, 4143 (2017).

${ }^{24}$ T. Bienville, L. Belliard, P. Siry, and B. Perrin, Superlattices Microstruct. 35, 363 (2004).

${ }^{25}$ T. Bienville, J. F. Robillard, L. Belliard, I. Roch-Jeune, A. Devos, and B. Perrin, Ultrasonics 44, 1289 (2006).

${ }^{26}$ A. Crut, P. Maioli, N. Del Fatti, and F. Vallée, Phys. Rep. 549, 1 (2014).

${ }^{27}$ L. Pochhammer, J. Reine Angew. Math. 81, 324 (1876).

${ }^{28}$ C. Chree, Trans. Camb. Philos. Soc. 14, 250 (1889).

${ }^{29}$ A. E. Armenàkas, D. C. Gazis, and G. Herrmann, Free Vibrations of Circular Cylindrical Shells (Pergamon Press Inc., 1969).

${ }^{30}$ D. Martínez-Gutiérrez, and V. R. Velasco, Physica E 54, 86 (2013).

${ }^{31}$ T. Haag, M.-I. Richard, U. Welzel, V. Favre-Nicolin, O. Balmes, G. Richter, E. J. Mittemeijer, and O. Thomas, Nano Lett. 13, 1883 (2013).

${ }^{32}$ H. Al-Zahrani, J. Pal, M. A Migliorato, G. Tse, and D. Yu, Nano Energy 14, 382 (2015).

${ }^{33}$ C. Leclere, T. W. Cornelius, Z. Ren, A. Davydok, J.-S. Micha, O. Robach, G. Richter, L. Belliard, and O. Thomas, J. Appl. Cryst. 48, 291 (2015). 